\title{
State-selective electron transfer and ionization in collisions of highly charged ions with ground-state $\mathrm{Na}(3 s)$ and laser-excited $\mathrm{Na}^{*}(3 \mathrm{p})$
}

\author{
I. Blank,${ }^{1}$ S. Otranto, ${ }^{2}$ C. Meinema, ${ }^{1}$ R. E. Olson,${ }^{3}$ and R. Hoekstra ${ }^{1}$ \\ ${ }^{1}$ KVI, Atomic Physics, Zernikelaan 25, NL-9747 AA, Groningen, The Netherlands \\ ${ }^{2}$ IFISUR and Departamento de Física, Universidad Nacional del Sur, 8000 Bahía Blanca, Argentina \\ ${ }^{3}$ Physics Department, Missouri University of Science and Technology, Rolla Missouri 65409, USA
}

(Received 26 October 2011; published 21 February 2012)

\begin{abstract}
Single electron transfer and ionization in collisions of $\mathrm{N}^{5+}$ and $\mathrm{Ne}^{8+}$ with ground state $\mathrm{Na}(3 s)$ and laser excited $\mathrm{Na}^{*}(3 p)$ are investigated both experimentally and theoretically at collision energies from 1 to $10 \mathrm{keV} / \mathrm{amu}$, which includes the classical orbital velocity of the valence electron. State-selective partial cross sections are obtained using recoil-ion momentum spectroscopy in combination with a magneto-optically cooled $\mathrm{Na}$ atom target. A strong dependence of the cross sections on the collision energy is observed. In general, both the relative magnitude and the energy dependence are found to be in good agreement with classical-trajectory Monte Carlo calculations.

DOI: 10.1103/PhysRevA.85.022712

PACS number(s): $34.70 .+\mathrm{e}$
\end{abstract}

\section{INTRODUCTION}

Single electron transfer is the dominant process in collisions of highly charged ions with atoms at low-keV/amu energies and therefore plays a significant role in the understanding of all plasma environments. In astrophysical processes such as the interaction of cometary atmospheres with the solar wind electron transfer gives rise to photon emission [1-3]. Furthermore, electron transfer processes play a crucial role in tokamak fusion plasma. For example, photon emission after electron transfer is used as a plasma diagnostics tool [4]. To enhance radiative cooling of the plasma in the diverter or edge layer of the tokamak, extrinsic impurities such as nitrogen and neon are puffed into the vessel [5,6]. Following injection, these impurity atoms are multiply ionized by the plasma. Collisions of the now highly charged impurities and hydrogen atoms present in the edge plasma cause electron transfer and subsequent photon emission. In the colder outer plasma regions electron transfer from metastable $\mathrm{H}^{*}(n=2)$ and $\mathrm{D}^{*}(n=2)$ to highly charged ions is a significant process due to the comparatively low ionization potential of the excited atoms [7,8].

Until now it is not feasible to perform charge transfer experiments with targets such as $\mathrm{H}^{*}(n=2)$ or $\mathrm{D}^{*}(n=2)$. It has been proposed that cross sections for these metastables can be interpolated from those for alkali-metal atoms since the ionization energies and thus the cross sections are similar $[9,10]$. In particular, the ionization potentials of $\mathrm{H}^{*}(n=2)$ and $\mathrm{D}^{*}(n=2)(3.40 \mathrm{eV})$ lie in between those of $\mathrm{Na}(3 s)$ $(5.14 \mathrm{eV})$ and $\mathrm{Na}^{*}(3 p)$ (3.04 eV). This makes these atoms likely candidates for interpolation of electron transfer cross sections of $\mathrm{H}^{*}(n=2)$ and $\mathrm{D}^{*}(n=2)$.

At low energies (i.e., when the projectile velocity is smaller than the classical orbital velocity of the target electron), electron transfer is known to be very selective with respect to the final state with usually one or two dominating channels. With increasing projectile energy the capture process leads to population of ever higher final states and a rise of the ionization cross section.

In a previous work experimental state-selective electron transfer and ionization cross sections in collisions of $\mathrm{O}^{6+}$ with ground state $\mathrm{Na}(3 s)$ have been compared with with theoretical results obtained with the close-coupling twocenter basis generator method (TC-BGM) [11]. Generally good agreement has been found between the experimental and theoretical results with respect to electron transfer. However, while relative ionization cross sections could be obtained experimentally the numerical accuracy of the theoretical calculations was not sufficient to yield results.

In this work we present experimental and theoretical stateselective cross sections for single electron transfer in collisions of highly charged $\mathrm{N}^{5+}$ and $\mathrm{Ne}^{8+}$ ions with ground-state $\mathrm{Na}(3 s)$ and laser-excited $\mathrm{Na}^{*}(3 p)$. Experimental differential cross sections are extracted from the $\mathrm{Na}^{+}$recoil spectra obtained using the MOTRIMS technique [12-14] which combines recoil ion momentum spectroscopy (RIMS) [15] with a target that is laser-cooled and trapped in a magneto-optical trap (MOT). The experimental data are compared with three-body classical-trajectory Monte Carlo (CTMC) calculations [16]. A big advantage of the CTMC method is that it is not limited to a finite basis set and is thus very applicable to electron transfer and ionization processes involving highly charged ions. The investigated energy range from 1 to $10 \mathrm{keV} / \mathrm{amu}$ contains collision velocities corresponding to the velocities of the target valence electron of $9.4 \mathrm{keV} / \mathrm{amu}$ for $\mathrm{Na}(3 s)$ and $5.6 \mathrm{keV} / \mathrm{amu}$ for $\mathrm{Na}^{*}(3 p)$. Since the relative ionization cross sections are also obtained experimentally and theoretical cross sections can be calculated in the CTMC frame work as well, results on ionization are included in this paper to complete the picture of the investigated collision processes. Finally, a scaling is proposed for interpolation of state-selective cross sections for electron transfer with a $\mathrm{H}^{*}(n=2)$ target based on the results obtained with $\mathrm{Na}(3 s)$ and $\mathrm{Na}^{*}(3 p)$.

\section{EXPERIMENTAL METHOD}

Our MOTRIMS apparatus has been described elsewhere [17]. In short, the ${ }^{23} \mathrm{Na}$ atoms providing the target are cooled and trapped in a magneto-optical trap (MOT) using a magnetic field with a gradient of $20 \mathrm{G} / \mathrm{cm}$ and three counterpropagating laser beams with a diameter of $20 \mathrm{~mm}$ each, which are red detuned to the $3 s^{2} S_{1 / 2}, F=2 \rightarrow 3 p^{2} P_{3 / 2}, F=3$ transition frequency by $20 \mathrm{MHz}$. An electro-optical modulator is used to 
create a frequency side band that serves as the repumper, red detuned to the $3 s^{2} S_{1 / 2}, F=1 \rightarrow 3 p^{2} P_{3 / 2}, F=2$ transition frequency to avoid population build up in the $F=1$ hyperfine level of the Na ground state. The total light intensity is about $100 \mathrm{~mW}$. The ion beam provided by our electron cyclotron resonance ion source is collimated to about $1 \mathrm{~mm}$ and crossed with the MOT. The resulting $\mathrm{Na}^{+}$recoil ions are extracted transverse to the ion-beam direction by a low electric field $(<0.5 \mathrm{~V} / \mathrm{cm})$ toward the detector where their two-dimensional position is recorded. From this data the recoil momentum vector can be reconstructed.

The $Q$-value of the process (i.e., the total change of binding energies of all electrons before and after the collision) is related to the longitudinal momentum of the recoil ions by

$$
p_{\text {long }}=\frac{Q}{v_{\mathrm{p}}}-\frac{1}{2} r v_{\mathrm{p}} .
$$

Here $v_{\mathrm{p}}$ is the projectile ion velocity and $r$ is the number of transferred electrons. Since the initial binding energies of the collision partners are well known, the $Q$-value spectrum directly yields the final state distribution of the electrons in the projectile. An example of a $Q$-value spectrum resulting from single electron transfer in collisions of $\mathrm{N}^{5+}$ with $\mathrm{Na}(3 s)$ at $2.5 \mathrm{keV} /$ amu collision energy is shown in Fig. 1(a). Relative cross sections are obtained by a least-squares fitting procedure. For capture from ground-state $\mathrm{Na}(3 s), Q$-values are assigned using the peaks corresponding to more strongly bound final states. These states have a larger energy spacing so that they are sufficiently separated to obtain a channel to $Q$-value conversion even at the higher collision energies. From the fit of the $Q$-value spectrum, the experimental momentum resolution is determined as $\Delta p_{\text {RMS }}=0.05$ a.u.. At $2.5 \mathrm{keV} /$ amu collision energy this corresponds to a $Q$-value resolution of $\Delta Q_{\mathrm{RMS}}=$ $0.43 \mathrm{eV}$. Using this conversion the peak positions for higher final states with smaller $Q$-value spacing have been fixed.

Even though electron transfer is the main process in the investigated collision-energy range, recoils resulting from ionization of the target are also recorded. In the $Q$-value spectra, ionization events appear at $Q \geqslant I$ where $I$ is the target's ionization potential. Taking the experimental resolution into account as described in [18], relative ionization cross sections can be obtained from the $Q$-value spectra as well.

During standard operation, the atomic sample in a MOT contains a mixture of ground-state and excited-state atoms. For the experiments with ground-state $\mathrm{Na}(3 s)$, all laser beams of the MOT are switched off using an acousto-optical modulator (AOM) with a frequency of about $7 \mathrm{kHz}$ and a duty cycle of $67 \%$. The measurements are performed with a continuous ion beam. Applying a timing gate on the detector synchronized to the AOM switching frequency ensures that all target atoms have decayed to the ground state before the collision. This is possible due to the much shorter decay time of the excited $\mathrm{Na}^{*}(3 p)$ with respect to the AOM switching frequency. In order to obtain spectra resulting from a target containing ground-state and excited-state atoms, the detector gate time is changed appropriately. All other experimental parameters are unchanged so that the total number of atoms in the MOT is essentially the same during both measurements. Since the atoms are excited by the six circularly polarized trapping laser

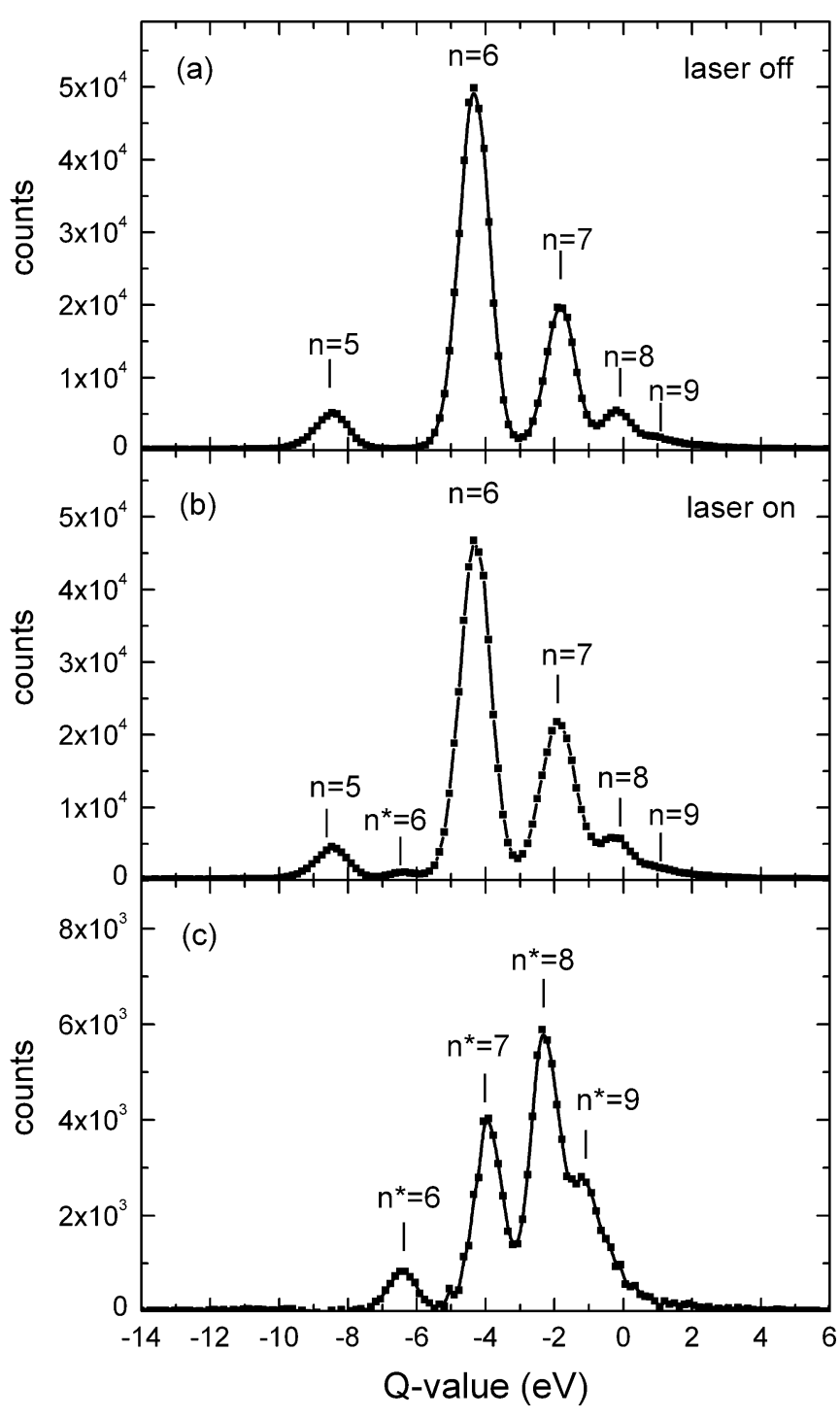

FIG. 1. $Q$-value spectrum obtained with $\mathrm{N}^{5+}$ projectiles at a collision energy of $2.5 \mathrm{keV} / \mathrm{amu}$ with (a) laser off and (b) laser on and (c) the resulting spectrum for $\mathrm{Na}^{*}(3 p)$.

beams of the MOT, the $\mathrm{Na}^{*}(3 p)$ atoms are unpolarized. A pure $\mathrm{Na}^{*}(3 p)$ spectrum is obtained via

$$
S^{*}=\frac{1}{f}\left[S^{\text {on }}-(1-f) S^{\text {off }}\right],
$$

where $S^{\text {off }}$ and $S^{\text {on }}$ are the recoil spectra in the case of the laser beams being switched off or on, respectively, normalized to the total amount of charge passing through the MOT, and $f$ is the excited-state fraction [i.e., the fraction of $\mathrm{Na}^{*}(3 p)$ atoms in the MOT sample]. One way of obtaining the excited-state fraction is to determine the decrease of one well-resolved and separated peak resulting from electron transfer from $\mathrm{Na}(3 s)$ [19,20]. Figures 1(a) and 1(b) show a $Q$-value spectrum resulting from collisions of $\mathrm{N}^{5+}$ at a collision energy of $2.5 \mathrm{keV} / \mathrm{amu}$ with the laser off and on, respectively. Here the final state $\mathrm{N}^{4+}(n=5)$ resulting from capture from ground state $\mathrm{Na}(3 s)$ and the final state $\mathrm{N}^{4+}\left(n^{*}=6\right)$ resulting from capture from excited-state $\mathrm{Na}^{*}(3 p)$ are well separated. However, with increasing collision energy the $Q$-value resolution decreases 
[cf. Eq. (1)] so that, for a target containing both $\mathrm{Na}(3 s)$ and $\mathrm{Na}^{*}(3 p)$, capture events resulting in $\mathrm{N}^{4+}(n=5)$ and $\mathrm{N}^{4+}\left(n^{*}=6\right)$ start to overlap in the $Q$-value spectrum. This makes it impossible to obtain the excited-state fraction from the decrease of the $\mathrm{N}^{4+}(n=5)$ peak, since the $\mathrm{N}^{4+}\left(n^{*}=6\right)$ peak does not increase proportionally. To obtain the relative cross sections for $\mathrm{Na}^{*}(3 p)$ at higher collision energies, the spectra $S^{\text {on }}$ with laser on have been fit with the fit function obtained from the pure $\mathrm{Na}(3 s)$ spectrum $S^{\text {off }}$ weighted with a fit parameter $p=(1-f)$ to account for the total decrease of events resulting from $\mathrm{Na}(3 s)$. The peak positions of the final states resulting from capture from $\mathrm{Na}^{*}(3 p)$ have been fixed using the same conversion as for a pure $\mathrm{Na}(3 s)$ spectrum and the peak heights were determined by a fit as well. This method has been tested at low collision energies where the excited-state fraction can also be obtained from one well-resolved peak resulting from capture from $\mathrm{Na}(3 s)$. The results obtained with the two methods differ by less than $1 \%$. Typical excitation fractions are between $10 \%$ and $20 \%$. The resulting $Q$-value spectrum for $\mathrm{Na}^{*}(3 p)$ is given in Fig. 1(c).

To obtain an estimate on the experimental uncertainties of the cross sections, two data sets have been measured for almost all energies. The results have been averaged to obtain the presented values. From this we find that the experimental uncertainties of the state-selective cross sections for capture from $\mathrm{Na}(3 s)$ are $5 \%$ for the well-resolved lower final states and $10 \%$ for the higher final states with smaller $Q$-value spacing. For electron capture from $\mathrm{Na}^{*}(3 p)$ the uncertainties for the relative experimental cross sections are $15 \%$. The uncertainties of the ionization cross sections are estimated at $25 \%$ due to the low event rate.

\section{THEORETICAL METHOD}

Theoretical single electron capture and ionization cross sections have been calculated using the CTMC method [16]. Hamilton's equations are solved for a mutually interacting three-body system. To mimic the experimental conditions, the center of mass of the $\mathrm{Na}$ target is frozen at the beginning of each simulation. The active electron evolves under the central potential model developed by Green et al. from Hartree-Fock calculations [21], and later generalized by Garvey et al. [22]:

$$
\begin{aligned}
& V(r)=\frac{(N-1)[1-\Omega(r)]-Z}{r}, \\
& \Omega(r)=\left[\left(\frac{\eta}{\xi}\right)\left(e^{\xi r}-1\right)+1\right]^{-1} .
\end{aligned}
$$

Here, $(N-1)$ is the number of electrons present in the ion and $\eta$ and $\xi$ are the screening parameters which have been tabulated for ions with $2 \leqslant Z \leqslant 54$.

This parametrization leads to a much more realistic description of the active electron dynamics so that the electron correctly "sees" a nuclear charge that varies from the asymptotic value of $1+$ to the nuclear charge $11+$ at the coalescence point. The CTMC method directly includes the ionization channel and is not limited by basis-set size for the prediction of capture to very high-lying excited states. Since the electron tends to be captured to high- $n$ values with minimal contributions from the $s, p$, and $d$ states, quantum defects play a minor role and the orbital energies for the captured electron are similar to those obtained with bare projectiles. We represent the captured electron-projectile interaction by a Coulomb potential where the projectile's asymptotic charge is considered. A classical number $n_{\mathrm{c}}$ is obtained from the binding energy $E_{\mathrm{p}}$ of the electron relative to the projectile by

$$
E_{\mathrm{p}}=-Z_{\mathrm{p}}^{2} /\left(2 n_{\mathrm{c}}^{2}\right),
$$

where $Z_{\mathrm{p}}$ is the charge of the projectile core. Then, $n_{\mathrm{c}}$ is related to the quantum number $n$ of the final state by the condition derived by Becker and McKellar [23]:

$$
[(n-1)(n-1 / 2) n]^{1 / 3} \leqslant n_{\mathrm{c}} \leqslant[n(n+1)(n+1 / 2)]^{1 / 3} .
$$

The cross section for a definite $n$ state is then given by

$$
\sigma_{n}=N(n) \pi b_{\max }^{2} / N_{\text {tot }},
$$

where $N(n)$ is the number of events of electron capture to the level $n$ and $N_{\text {tot }}$ is the total number of trajectories integrated. The impact parameter $b_{\max }$ is the value beyond which the probability of electron capture is negligibly small.

\section{RESULTS AND DISCUSSION}

\section{A. Electron capture from ground-state sodium}

Since the overlap between the ion beam and the MOT cloud has not been determined during the measurements, only relative cross sections are obtained experimentally. We have used the theoretical total cross sections given in Table I to put the experimental data on an absolute scale. In the investigated collision-energy range the total cross sections show an almost linear decrease with increasing collision energy. Cross sections for capture by $\mathrm{N}^{5+}$ projectiles from $\mathrm{Na}(3 s)$ are in the order of 2 to $3 \times 10^{-14} \mathrm{~cm}^{2}$. In comparison, cross sections for capture by $\mathrm{Ne}^{8+}$ are about $60 \%$ larger, which directly reflects the higher projectile charge state.

Figure 2 shows state-selective experimental and theoretical capture cross sections for $\mathrm{N}^{5+}+\mathrm{Na}(3 s)$ collisions in the energy range between 1 and $10 \mathrm{keV} / \mathrm{amu}$. State-selective cross sections could be obtained for the final states $n=5$ to $n=9$, for population of higher excited states $n \geqslant 10$ a summed cross section is given. At collision energies below $7 \mathrm{keV} / \mathrm{amu}$, single electron capture preferentially populates the $\mathrm{N}^{4+}(n=6)$ final state with a cross section of $2.1 \times$ $10^{-14} \mathrm{~cm}^{2}$ at a collision energy of $1.8 \mathrm{keV} / \mathrm{amu}$. However,

TABLE I. Total CTMC cross sections for single electron capture in collisions of $\mathrm{N}^{5+}$ and $\mathrm{Ne}^{8+}$ with $\mathrm{Na}(3 s)$ and $\mathrm{Na}^{*}(3 p)$.

\begin{tabular}{lcccc}
\hline \hline $\begin{array}{l}\text { Projectile } \\
\text { Target }\end{array}$ & $\begin{array}{c}\mathrm{N}^{5+} \\
\mathrm{Na}(3 s)\end{array}$ & $\begin{array}{c}\mathrm{Ne}^{8+} \\
\mathrm{Na}(3 s)\end{array}$ & $\begin{array}{c}\mathrm{N}^{5+} \\
\mathrm{Na}(3 p)\end{array}$ & $\begin{array}{c}\mathrm{Ne}^{8+} \\
\mathrm{Na}(3 p)\end{array}$ \\
\hline$E(\mathrm{keV} / \mathrm{amu})$ & & \multicolumn{2}{c}{$\sigma\left(10^{-14} \mathrm{~cm}^{2}\right)$} & \\
1 & 2.91 & 4.96 & 8.35 & 14.3 \\
2 & 2.86 & 4.83 & 8.17 & 13.7 \\
3 & 2.78 & 4.71 & 7.69 & 13.0 \\
5 & 2.61 & 4.31 & 6.90 & 11.8 \\
7 & 2.44 & 4.10 & 6.13 & 10.9 \\
8.5 & 2.32 & 3.97 & 5.58 & 10.1 \\
10 & 2.20 & 3.53 & 4.87 & 9.48 \\
\hline \hline
\end{tabular}



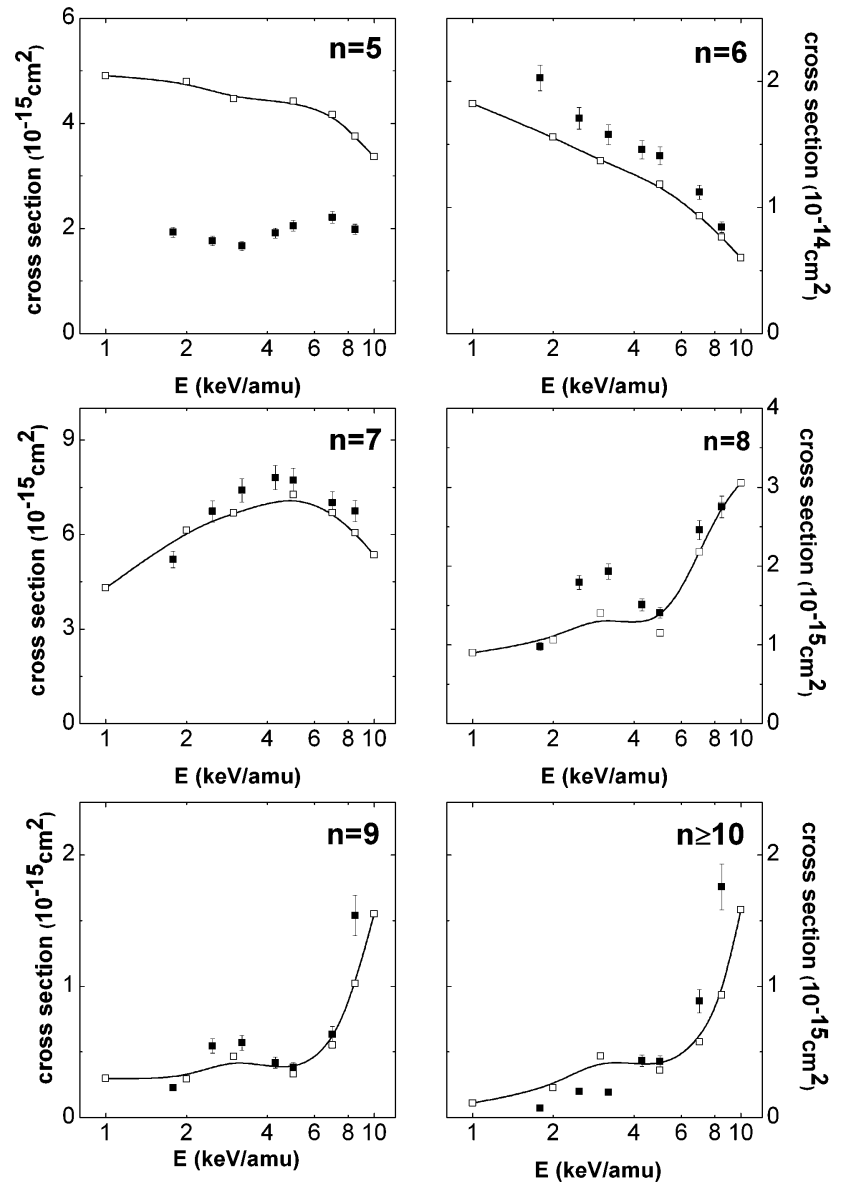

FIG. 2. Single electron cross sections for $\mathrm{N}^{5+}+\mathrm{Na}(3 s) \rightarrow$ $\mathrm{N}^{4+}(n)+\mathrm{Na}^{+}$collisions as a function of the collision energy. Full squares are for experiment, open squares are for CTMC. A $B$-spline line is drawn for the CTMC results to guide the eye.

with increasing collision energy the probability for capture into the $n=6$ state decreases in favor of the $n=7$ final state. The cross section for $n=7$ shows a distinct maximum at a collision energy of $4.3 \mathrm{keV} / \mathrm{amu}$ with $7.7 \times 10^{-14} \mathrm{~cm}^{2}$. The theoretical calculations give a similar behavior with increasing energy; however, the cross section peaks at a slightly higher energy of $5 \mathrm{keV} / \mathrm{amu}$. The cross sections for higher excited states $n=8, n=9$ and, $n \geqslant 10$ are below $5 \times 10^{-15} \mathrm{~cm}^{2}$ at energies below $6 \mathrm{keV} / \mathrm{amu}$ where they show a weak oscillatory structure maximizing around $3 \mathrm{keV} / \mathrm{amu}$, which is predicted by the theory as well. Above an energy of $6 \mathrm{keV} / \mathrm{amu}$, the cross sections for higher excited states $n \geqslant 8$ show a steep increase.

The only significant discrepancy of experiment and theory is observed in the case of the lowest and weakly populated final state $n=5$ where the CTMC calculations overestimate the relative cross section by a factor of two over the whole collision-energy range. We attribute this discrepancy to the neglect of multiple capture channels in the theoretical description. Double capture dominates at small impact parameters and will deplete flux from single capture to the low- $n$ levels. The calculated transition probabilities for $5.36 \mathrm{keV} / \mathrm{amu}$ are illustrated in terms of their impact-parameter dependence in Fig. 3. The $n=5$ final state is predominantly populated at

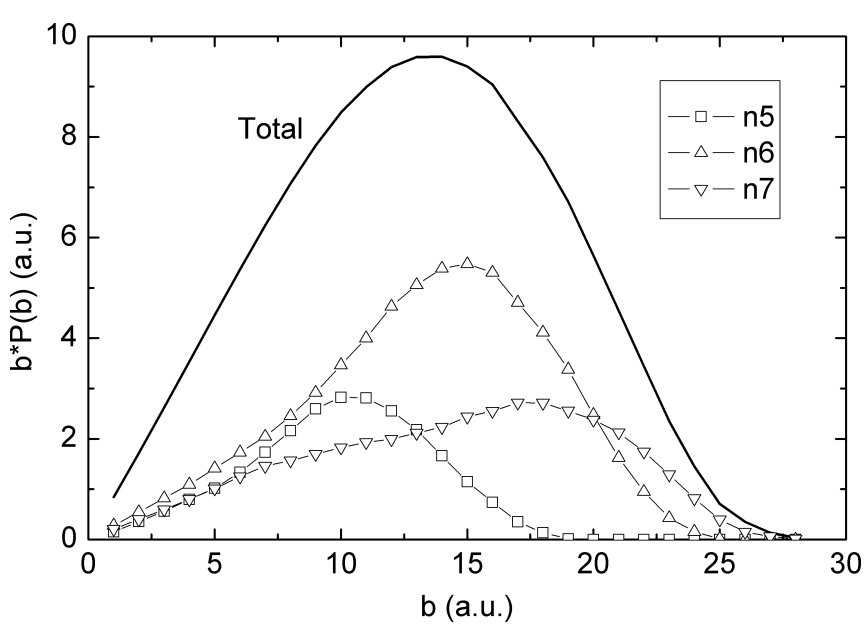

FIG. 3. Theoretical electron capture transition probabilities as a function of the impact parameter $b$ for product final states $n=5,6$, and 7 for collisions of $\mathrm{N}^{5+}$ with $\mathrm{Na}(3 s)$ at $5.36 \mathrm{keV} / \mathrm{amu}$.

small impact parameters so that the aforementioned effect plays a major role.

State-selective capture cross sections for collisions of $\mathrm{Ne}^{8+}$ with $\mathrm{Na}(3 s)$ are depicted in Fig. 4. The main capture channels lead to population of the $\mathrm{Ne}^{7+}(n=8)$ and $\mathrm{Ne}^{7+}(n=9)$ shells with a cross section of $1.5 \times 10^{-14} \mathrm{~cm}^{2}$ and $2.4 \times 10^{-14} \mathrm{~cm}^{2}$, respectively, at a collision energy of $2 \mathrm{keV} / \mathrm{amu}$. Both cross sections decrease with increasing energy. The cross sections for the $n=10$ final states show a rather weak dependence on the collision energy within the studied range and exhibit a slight oscillatory behavior. The opening up of more channels with increasing collision energy is a general feature of electron transfer, so that, similar to the $\mathrm{N}^{5+}$ case, a steep increase of the cross sections for population of highly excited final states $n=11$ and $n \geqslant 12$ is observed. This trend is also seen in the CTMC results whereas the theoretical calculations underestimate the experimental results by a factor of 1.7 at the highest collision energy. The cross section of the lowest significantly populated final state $n=7$ has a maximum at a collision energy of $7 \mathrm{keV} / \mathrm{amu}$. The theoretical results follow this trend; however, similar to the case of $\mathrm{N}^{5+}$, theory overestimates the cross sections by a factor of about two.

\section{B. Electron capture from excited-state sodium}

In collisions with excited $\mathrm{Na}^{*}(3 p)$ the lower binding energy of the valence electron manifests itself in large total cross sections, almost a factor three larger than for $\mathrm{Na}(3 s)$ [cf. Table I]. Furthermore, the main capture channels show a clear shift to higher final states.

For electron capture from excited $\mathrm{Na}^{*}(3 p)$ by $\mathrm{N}^{5+}$ stateselective cross sections are shown in Fig. 5 for final states $\mathrm{N}^{4+}(n=6)$ to $\mathrm{N}^{4+}(n=8)$ and a summed cross section is given for states with $n \geqslant 9$. At a collision energy of $1.8 \mathrm{keV} / \mathrm{amu}$ the main capture channel is $n=8$ with a cross section of $3.2 \times 10^{-14} \mathrm{~cm}^{2}$. At this energy the cross section for $n=7$ is only slightly lower with $2.7 \times 10^{-14} \mathrm{~cm}^{2}$. Capture into both states decreases rapidly with increasing collision energy in favor of more highly excited final states $n \geqslant 9$. However, the increase of the cross sections into very highly excited 

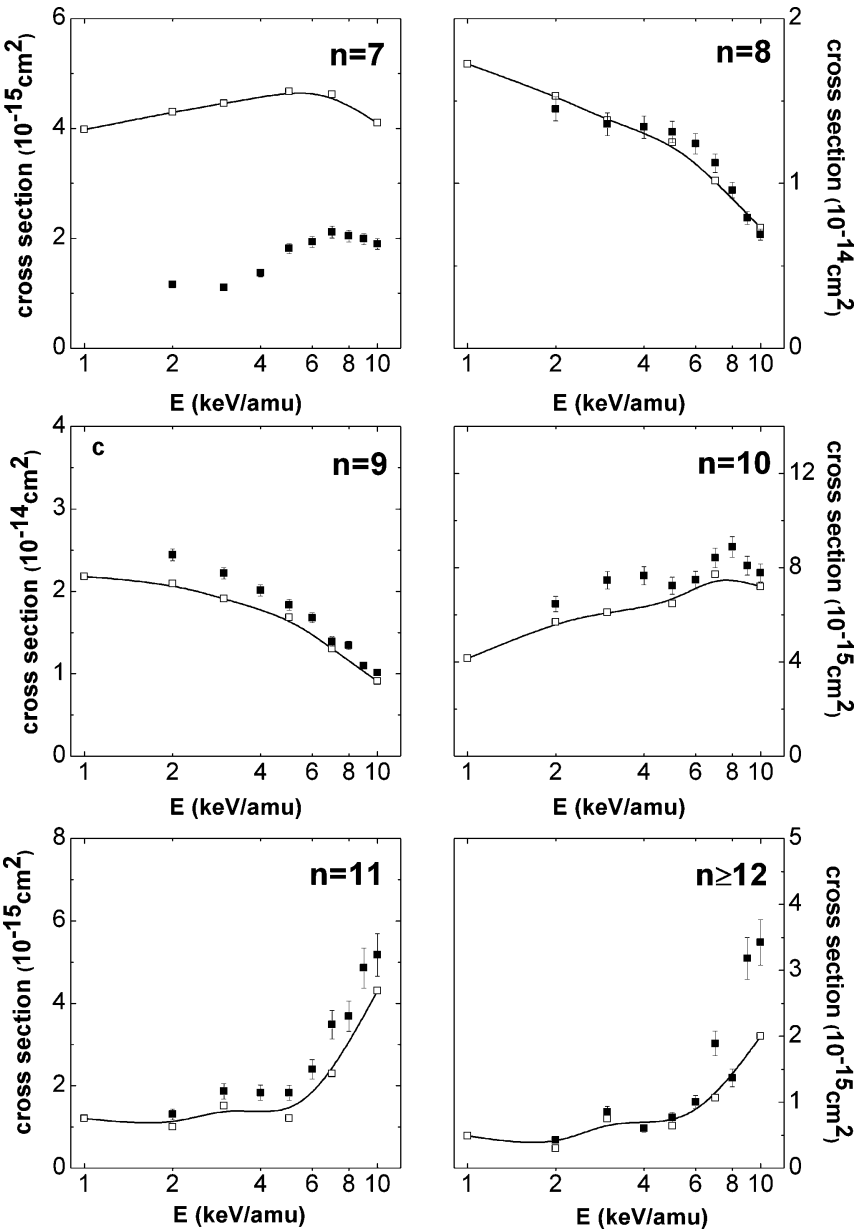

FIG. 4. Single electron cross sections for $\mathrm{Ne}^{8+}+\mathrm{Na}(3 s) \rightarrow$ $\mathrm{Ne}^{7+}(n)+\mathrm{Na}^{+}$collisions as a function of the collision energy. Full squares are for experiment, open squares are for CTMC. A $B$-spline line is drawn for the CTMC results to guide the eye.

states with increasing collision energy is less steep than with a ground-state $\mathrm{Na}(3 s)$ target, and the cross section even decreases above $8 \mathrm{keV} / \mathrm{amu}$. This trend is also seen in the CTMC results. The summed cross section for final states $n \geqslant 9$ has a maximum at a collision energy of around $7.5 \mathrm{keV} / \mathrm{amu}$ which is also observed in the theory. The cross section for the lowest significantly populated final state $n=6$ has a maximum at a collision energy around $4.3 \mathrm{keV} / \mathrm{amu}$. The theoretical result shows a similar dependence on collision energy; however, it is less pronounced than in the experimental data.

For $\mathrm{Ne}^{8+}$ projectiles final states $\mathrm{Ne}^{7+}(n=8)$ to $\mathrm{Ne}^{7+}(n=$ 12) could be resolved and a summed cross section is given for final states $\mathrm{Ne}^{7+}(n \geqslant 13)$. The state-selective cross section are shown in Fig. 6. At the lowest experimental collision energy of $2 \mathrm{keV} / \mathrm{amu}$ the main capture channel is $n=11$ with a cross section of $4.3 \times 10^{-14} \mathrm{~cm}^{2}$. This cross section decreases rapidly with increasing collision energy and is in excellent agreement with the theoretical result. A similar trend is observed with the neighboring final states $n=10$ and $n=12$. The cross section for the highest final states $n \geqslant 13$ increases with higher collision energies; however, the increase is less rapid than with ground-state $\mathrm{Na}(3 s)$ and the cross sections level off at $10 \mathrm{keV} / \mathrm{amu}$. The cross sections of the
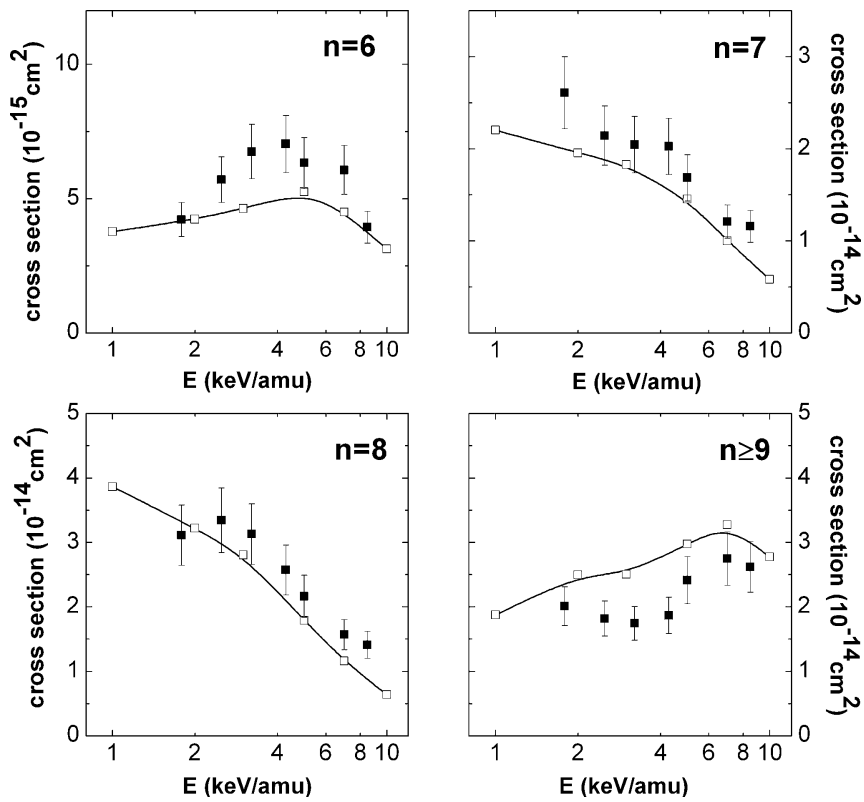

FIG. 5. Single electron cross sections for $\mathrm{N}^{5+}+\mathrm{Na}^{*}(3 p) \rightarrow$ $\mathrm{N}^{4+}(n)+\mathrm{Na}^{+}$collisions as a function of the collision energy. Full squares are for experiment, open squares are for CTMC. A $B$-spline line is drawn for the CTMC results to guide the eye.

lowest final states $n=8$ and $n=9$ show a maximum around 7 and $5 \mathrm{keV} / \mathrm{amu}$, respectively. While the relative ratio of the $n=9$ final state is well reproduced by the theory, the relative cross section for $n=8$ is underestimated by a factor of two.

\section{Ionization of ground- and excited-state sodium}

Although electron capture is by far the dominant process in the low-keV/amu energy range, ionization of the target becomes relevant at higher collision energies above the classical orbital velocity of the valence electron. Figure 7 shows relative experimental and theoretical ionization cross sections with respect to all single-electron removal events in collisions of $\mathrm{N}^{5+}$ and $\mathrm{Ne}^{8+}$ with $\mathrm{Na}(3 s)$ and $\mathrm{Na}^{*}(3 p)$. Due to the small ionization cross sections at low collision energies, experimental results could be obtained only for collision energies above $4.3 \mathrm{keV} / \mathrm{amu}$ for $\mathrm{N}^{5+}$ projectiles and $6 \mathrm{keV} / \mathrm{amu}$ for $\mathrm{Ne}^{8+}$ projectiles. Comparing the ionization cross section for $\mathrm{N}^{5+}$ and $\mathrm{Ne}^{8+}$, the cross section is lower for $\mathrm{Ne}^{8+}$ since, with increasing projectile charge, electron capture is favored over ionization. The relative ionization cross section of excited $\mathrm{Na}^{*}(3 p)$ is almost an order of magnitude larger than for ground-state $\mathrm{Na}(3 s)$ due to the lower binding energy.

Ionization rapidly increases with higher collision energy. In the case of an excited $\mathrm{Na}^{*}(3 p)$ target, the leveling off of capture into the highest final states can be attributed to the steep rise of the ionization cross sections at collision energies above the classical velocity of the valence electron. For the system $\mathrm{N}^{5+}+$ $\mathrm{Na}^{*}(3 p)$, ionization constitutes over $10 \%$ of the total electron removal cross section at the highest experimental energy of $8.5 \mathrm{kev} / \mathrm{amu}$. The CTMC results are in good agreement with experiment. To give more insight into the process, CTMC ionization cross sections have been calculated up to a collision energy of $25 \mathrm{keV} / \mathrm{amu}$. In this energy range, ionization is a significant contribution to total electron removal for a 

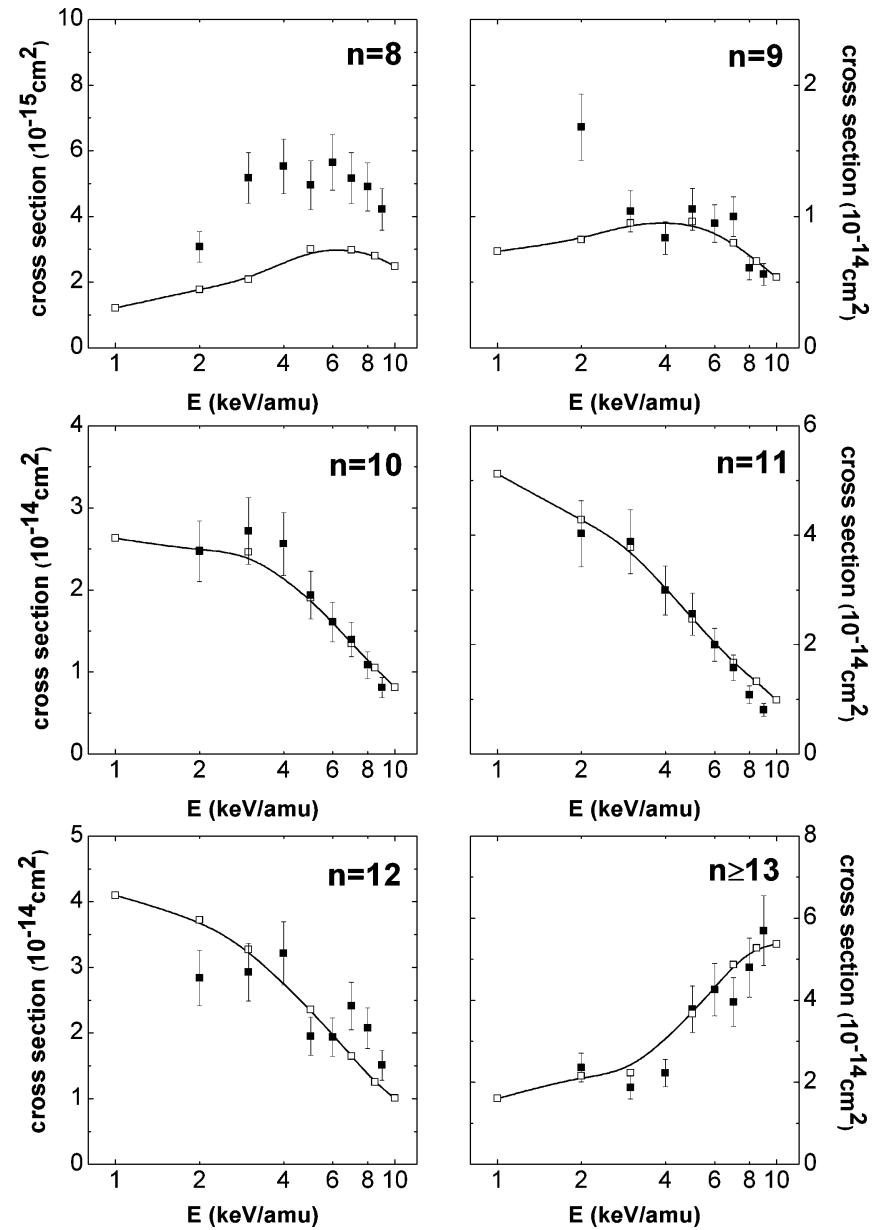

FIG. 6. Single electron cross sections for $\mathrm{Ne}^{8+}+\mathrm{Na}^{*}(3 p) \rightarrow$ $\mathrm{Ne}^{7+}(n)+\mathrm{Na}^{+}$collisions as a function of the collision energy. Full squares are for experiment, open squares are for CTMC. A $B$-spline line is drawn for the CTMC results to guide the eye.

ground-state $\mathrm{Na}(3 s)$ target and accounts for $30 \%$ of the total electron loss at $25 \mathrm{keV} / \mathrm{amu}$. For excited $\mathrm{Na}^{*}(3 p)$, ionization dominates the reaction dynamics and, at $25 \mathrm{keV} / \mathrm{amu}$, amounts to over $70 \%$ of the total electron removal.

In the low- to intermediate-energy range, a scaling law has been obtained by Wu et al. [24,25] for ionization in collisions of highly charged ions with $\mathrm{H}$ and $\mathrm{He}$ targets using generalized reduced cross sections $\widetilde{\sigma}=\sigma I^{1.3} / q$ as a function of a reduced collision energy $\widetilde{E}=E / I q^{1 / 2}$. While the energy dependence of the scaled cross sections for the systems studied in the present work are similar to the proposed scaling law, the absolute magnitude is underestimated by almost an order of magnitude. This is in line with our previous work [18] where it has been shown that this scaling law cannot simply be extended to weakly bound targets like $\mathrm{Na}(3 s)$ and $\mathrm{Na}^{*}(3 p)$.

\section{Scaling properties of cross sections}

To ascertain the possible applicability of the cross section obtained with $\mathrm{Na}(3 s)$ and $\mathrm{Na}^{*}(3 p)$ for interpolation of cross sections for $\mathrm{H}^{*}(n=2)$ scaling properties are discussed. Since the ionization potential of $\mathrm{Na}(3 s)$ is $5.14 \mathrm{eV}$ and that of $\mathrm{Na}^{*}(3 p)$ is $3.04 \mathrm{eV}$, it seems reasonable to expect that these systems should give insight into the $\mathrm{H}^{*}(n=2)$ target
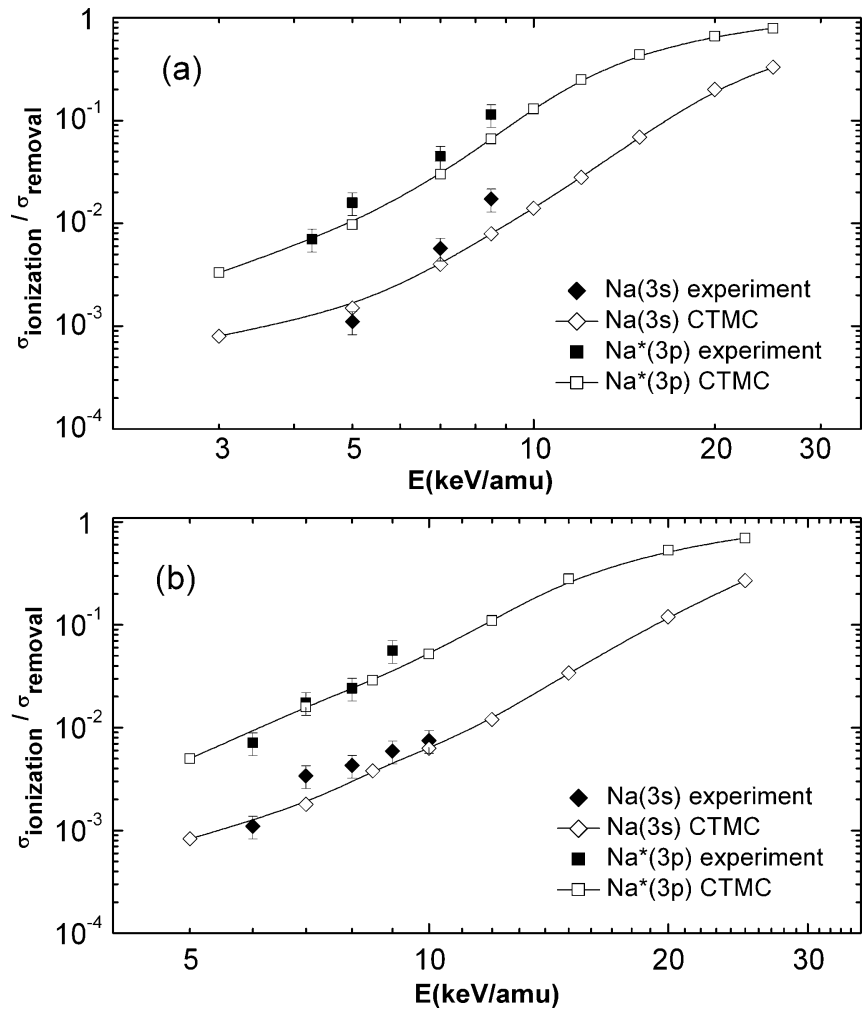

FIG. 7. Relative single-ionization cross sections in collisions of (a) $\mathrm{N}^{5+}$ and (b) $\mathrm{Ne}^{8+}$ with $\mathrm{Na}(3 s)$ and $\mathrm{Na}^{*}(3 p)$. A $B$-spline line is drawn for the CTMC results to guide the eye.

whose ionization potential is $3.40 \mathrm{eV}$. The latter system is experimentally inaccessible, but its state-selective cross sections are much needed for modeling of nuclear fusion reactors.

Figure 8 shows CTMC cross sections as a function of the final $n$ state for collisions of $5 \mathrm{keV} / \mathrm{amu} \mathrm{Ne}^{8+}$ with $\mathrm{Na}(3 s), \mathrm{Na}^{*}(3 p)$, and $\mathrm{H}^{*}(n=2)$ targets. From the ionization potentials, one would assume that the cross sections of $\mathrm{H}^{*}(n=$ 2) are closer to $\mathrm{Na}^{*}(3 p)$ than $\mathrm{Na}(3 s)$. However, from Fig. 8 it is seen that the $\mathrm{H}^{*}(n=2)$ results are more similar to $\mathrm{Na}(3 s)$

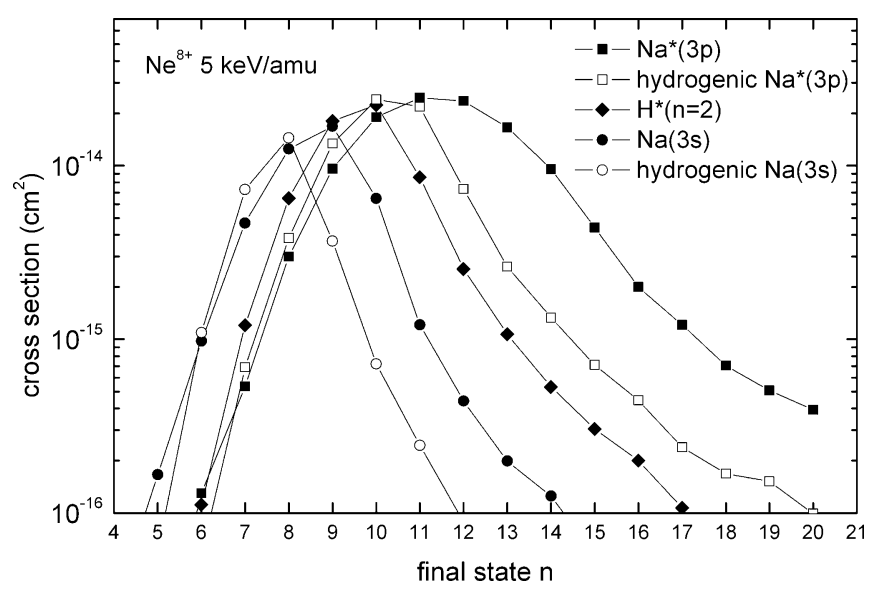

FIG. 8. State-selective CTMC cross sections as a function of the final state $n$ for collisions of $5 \mathrm{keV} / \mathrm{amu} \mathrm{Ne}^{8+}$ with $\mathrm{Na}(3 s), \mathrm{Na}^{*}(3 p)$, $\mathrm{H}^{*}(n=2)$, and hydrogenic $\mathrm{Na}(3 s)$ and $\mathrm{Na}^{*}(3 p)$. Straight lines are drawn to guide the eye. 


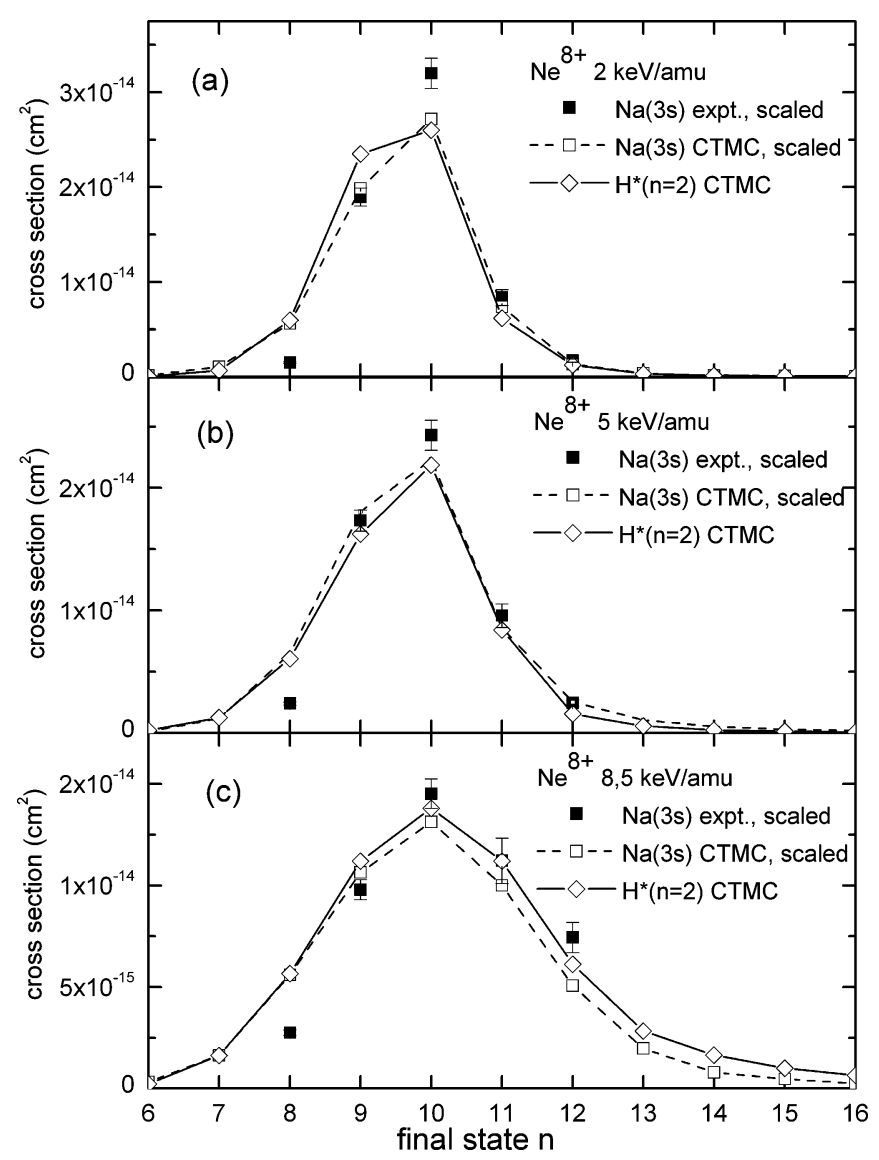

FIG. 9. State-selective CTMC cross sections as a function of the final state $n$ for collisions of $\mathrm{Ne}^{8+}$ with $\mathrm{H}^{*}(n=2)$ compared with scaled experimental and CTMC cross sections for collisions of $\mathrm{Ne}^{8+}$ with $\mathrm{Na}(3 s$ ) at (a) $2 \mathrm{keV} / \mathrm{amu}$, (b) $5 \mathrm{keV} / \mathrm{amu}$, and (c) $8.5 \mathrm{keV} / \mathrm{amu}$. Straight lines are drawn through the CTMC results to guide the eye.

than $\mathrm{Na}^{*}(3 p)$. This at first contradicts the assumption that cross sections scale with the ionization potential of the target. Figure 8 also shows CTMC cross sections for "hydrogenic" $\mathrm{Na}(3 s)$ and $\mathrm{Na}^{*}(3 p)$; that is, assuming a target atom with the ionization potential of $\mathrm{Na}(3 s)$ or $\mathrm{Na}^{*}(3 p)$ but a hydrogenic $1 / R$ potential. Here the cross sections for $\mathrm{H}^{*}(n=2)$ are closer to "hydrogenic" $\mathrm{Na}^{*}(3 p)$, as one would expect. This behavior can be explained by the nonhydrogenic character of the $\mathrm{Na}$ potential as the screening of the nuclear charge by the inner shell electrons as seen by the valence electron depends strongly on the distance to the nucleus.

A scaling law for electron capture in collisions of $\mathrm{A}^{q+}$ with $\mathrm{H}(n=1)$ for projectile charge states $q=1$ to 20 has been proposed in Ref. [26]. This scaling law predicts that the the $n$ value at which the state-selective cross section is maximum should be located at

$$
n_{m}=n_{i} q^{3 / 4}=\left(I_{H} / I_{T}\right)^{1 / 2} q^{3 / 4}
$$

where $n_{i}$ is related to the ionization potential of ground-state atomic hydrogen $I_{H}=13.6 \mathrm{eV}$ and the ionization potential of the target atom $I_{T}$ by simple hydrogenic scaling laws. More recent work on ground and excited $\mathrm{Li}$ and $\mathrm{Na}$ targets [10,27] indicates that the relationship given by Eq. (7) should be modified by a prefactor of 1.2 for alkali-metal targets. This is due to the nonhydrogenic potential that the active electron is subjected to in the alkali metals.

To explore this point, we have calculated the cross sections for the $\mathrm{H}^{*}(n=2)$ target with $\mathrm{N}^{5+}$ and $\mathrm{Ne}^{8+}$ projectiles in the 1 to $10 \mathrm{keV} / \mathrm{amu}$ energy range. From Fig. 8 and Eq. (7), we find that, to simulate a $\mathrm{H}^{*}(n=2)$ target, the position of the maximum $n$ level for $\mathrm{Na}(3 s)$ should be moved up by one $n$ unit. To obtain a quantitative agreement as well, the magnitude of the $\mathrm{Na}(3 s)$ cross sections is increased by a factor of 1.3. The comparison of the scaled experimental and CTMC results obtained with $\mathrm{Na}(3 s)$ and the CTMC results with $\mathrm{H}^{*}(n=2)$ for impact energies of 2, 5, and $8.5 \mathrm{keV} / \mathrm{amu}$ are shown in Fig. 9. Although the agreement is not perfect, it provides a good first estimate for the $\mathrm{H}^{*}(n=2)$ state-selective cross sections.

\section{CONCLUSIONS}

In this work, experimental and theoretical state-selective single electron capture and ionization cross sections have been presented for highly charged ions colliding with ground- and excited-state sodium atoms. The investigated energy ranges from 1 to $10 \mathrm{keV} / \mathrm{amu}$, which includes the velocity matching point of the projectile velocity and the classical orbital velocity of the target electron. The partial cross sections show a distinct dependence on the collision energy. As a general trend, the cross section of the dominant channel decreases with increasing energy in favor of higher excited final states and ionization of the target. In general, good agreement is found between experiment and the CTMC calculations. It is notable that theory follows the population of the high- $n$ levels after capture as the collision energy is increased and the ionization channel rises in importance. The only failure of theory presents itself for the prediction of the low- $n$ levels with the ground-state $\mathrm{Na}(3 s)$ target. We ascribe this discrepancy as due to the neglect of multiple capture in the theoretical description. We will investigate this point further by experimentally determining the states produced after multiple capture and theoretically implementing an $n$-body approach that includes the $L$-shell electrons on the sodium atom. Further work will also include transverse momentum spectra so that we can test theory at the impact-parameter level.

A simple scaling to interpolate state-selective electron capture cross sections for $\mathrm{H}^{*}(n=2)$ targets from data obtained with $\mathrm{Na}(3 s)$ is proposed. This scaling is tested using CTMC cross sections calculated for collisions of $\mathrm{Ne}^{8+}$ and $\mathrm{N}^{5+}$ with $\mathrm{H}^{*}(n=2)$ and $\mathrm{Na}(3 s)$. Although not in perfect agreement, the data for $\mathrm{Na}(3 s)$ provides a good first estimate of the $\mathrm{H}^{*}(n=2)$ state-selective cross sections. More work is needed in order to define a scaling applicable to a wide range of collision systems.

\section{ACKNOWLEDGMENTS}

Work at KVI is sponsored by the Helmholtzzentrum für Schwerionenforschung GmbH (GSI), Germany - KVI University of Groningen collaboration agreement. Work at UNS supported by PGI 24/F049, PICT-2007-00887 of the ANPCyT and PIP 112-200801-02760 of CONICET (Argentina). 
[1] T. E. Cravens, Geophys. Res. Lett. 24, 105 (1997).

[2] D. Bodewits, D. J. Christian, M. Torney, M. Dryer, C. M. Lisse, K. Dennerl, T. H. Zurbuchen, S. J. Wolk, A. G. G. M. Tielens, and R. Hoekstra, Astron. Astrophys. 469, 1183 (2007).

[3] S. Otranto, R. E. Olson, and P. Beiersdorfer, J. Phys. B 40, 1755 (2007).

[4] R. C. Isler, Plasma Phys. Controlled Fusion 36, 171 (1994).

[5] J. Rapp, M. R. de Baar, W. Fundamenski, M. Brix, R. Felton, C. Giroud, A. Huber, S. Jachmich, E. Joffrin, I. Nunes, G. J. van Rooij, M. Stamp, G. Telesca, R. Zagorski, and JET EFDA contributors, J. Nucl. Mater. 390-391, 238 (2009).

[6] G. Telesca, U. Samm, B. Unterberg, R. Koch, A. M. Messiaen, and G. V. Oost, Nucl. Fusion 36, 347 (1996).

[7] R. Hoekstra, H. Anderson, F. W. Bliek, M. von Hellermann, C. F. Maggi, R. E. Olson, and H. P. Summers, Plasma Phys. Controlled Fusion 40, 1541 (1998).

[8] H. Anderson, M. von Hellermann, R. Hoekstra, L. D. Horton, A. C. Howman, R. W. T. Konig, R. Martin, R. E. Olson, and H. P. Summers, Plasma Phys. Controlled Fusion 40, 781 (2000).

[9] F. W. Bliek, H. O. Folkerts, R. Morgenstern, R. Hoekstra, L. Meng, R. E. Olson, W. Fritsch, M. von Hellermann, and H. P. Summers, Nucl. Instrum. Methods B 98, 195 (1995).

[10] S. Otranto and R. E. Olson, J. Phys. B 43, 155203 (2010).

[11] S. Knoop, M. Keim, H. J. Lüdde, T. Kirchner, R. Morgenstern, and R. Hoekstra, J. Phys. B 38, 3163 (2005).

[12] M. van der Poel, C. V. Nielsen, M.-A. Gearba, and N. Andersen, Phys. Rev. Lett. 87, 123201 (2001).

[13] J. W. Turkstra, R. Hoekstra, S. Knoop, D. Meyer, R. Morgenstern, and R. E. Olson, Phys. Rev. Lett. 87, 123202 (2001).
[14] X. Fléchard, H. Nguyen, E. Wells, I. Ben-Itzhak, and B. D. DePaola, Phys. Rev. Lett. 87, 123203 (2001).

[15] J. Ullrich, R. Moshammer, A. Dorn, R. Dörner, L. P. H. Schmidt, and H. Schmidt-Böcking, Rep. Prog. Phys. 66, 1463 (2003).

[16] R. E. Olson and A. Salop, Phys. Rev. A 16, 531 (1977).

[17] S. Knoop, R. E. Olson, H. Ott, V. G. Hasan, R. Morgenstern, and R. Hoekstra, J. Phys. B 38, 1987 (2005).

[18] S. Knoop, V. G. Hasan, H. Ott, R. Morgenstern, and R. Hoekstra, J. Phys. B 39, 2021 (2006).

[19] F. Aumayr, M. Gieler, J. Schweinzer, H. Winter, and J. P. Hansen, Phys. Rev. Lett. 68, 3277 (1992).

[20] M. Gieler, F. Aumayr, J. Schweinzer, W. Koppensteiner, W. Husinsky, H. P. Winter, K. Lozhkin, and J. P. Hansen, J. Phys. B 26, 2137 (1993).

[21] A. E. S. Green, D. L. Sellin, and A. S. Zachor, Phys. Rev. 184, 1 (1969).

[22] R. H. Garvey, C. H. Jackman, and A. E. S. Green, Phys. Rev. A 12, 1144 (1975).

[23] R. L. Becker and A. D. McKellar, J. Phys. B 17, 3923 (1984).

[24] W. Wu, C. L. Cocke, J. P. Giese, F. Melchert, M. L. A. Raphaelian, and M. Stöckli, Phys. Rev. Lett. 75, 1054 (1995).

[25] W. Wu, E. F. Deveney, S. Datz, D. D. Desai, H. F. Krause, J. M. Sanders, C. R. Vane, C. L. Cocke, and J. P. Giese, Phys. Rev. A 53, 2367 (1996).

[26] R. E. Olson, Phys. Rev. A 24, 1726 (1981).

[27] K. R. Cornelius, K. Wojtkowski, and R. E. Olson, J. Phys. B 33, 2017 (2000). 